\title{
A case of a single intracranial vertebral artery and cerebral infarct
}

\author{
K. Blackburn, L. Labranche, J. Kalmey, R. Kulesza \\ Department of Anatomy, Lake Erie College of Osteopathic Medicine, Erie, PA, United States
}

[Received: 25 November 2015; Accepted: 4 January 2016]

\begin{abstract}
The vertebral arteries are commonly affected by anatomical variation. This variation ranges from slight asymmetry in arterial diameter between the right and left sides to complete absence of a vertebral artery on one side. Asymmetry in diameter is a common observation, although complete absence of the artery is rare. Herein, we report on a 79-year-old male anatomical donor who, upon brain removal, was found to have a single intracranial vertebral artery which was the sole source of the basilar artery. During dissection of the neck, both right and left vertebral arteries were identified arising from the subclavian arteries. The vertebral arteries were dissected from the transverse foramina and followed into the skull. The right vertebral artery terminated by supplying the spinal cord, consistent with the distribution of the posterior spinal artery. Such vascular anomalies are clinically significant, as they may lead to abnormal patterns of sensory-motor deficiencies in stroke and are at risk of iatrogenic injury during surgical procedures. (Folia Morphol 2017; 76, 1: 134-138)
\end{abstract}

Key words: anatomical variation, basilar artery, congenital anomaly

\section{INTRODUCTION}

The vertebral artery (VA) arises bilaterally from the first segment of the subclavian artery at the root of the neck, and passes superior and posteriorly in the interval between the vertical part of the longus colli and anterior scalene muscles to the level of the $6^{\text {th }}$ cervical vertebra. The VA passes superiorly through the transverse foramen of the $6^{\text {th }}$ cervical vertebra and all subsequent transverse foramina. After exiting the transverse foramen of the atlas, the VA turns posteromedially and courses along the transverse plane, in a groove along the posterior arch of the atlas. From this position, the VA turns anteriorly to enter the vertebral canal, pierces the dura mater and traverses the foramen magnum. Once inside the posterior cranial fossa, the VA courses superiorly along the anterior surface of the medulla oblongata to the inferior aspect of the pons. Here, it joins the VA from the contralateral side, in the midline, to form the basilar artery. The basilar artery courses along the anterior surface of the pons and upon reaching the midbrain, divides into left and right posterior cerebral arteries, which anastomose with the internal carotid arteries via the posterior communicating arteries. Together, the VAs supply deep neck muscles, spinal cord and provide the posterior circulation to the brain supplying the brainstem, cerebellum, thalamus, occipital lobe and temporal lobe of the cerebral cortex.

The VAs are commonly asymmetric in diameter, with the left most often having a larger diameter than the right $[13,15]$. Beyond this, the VA is subject to little anatomical variation (estimated to be $\sim 5 \%$ of subjects). The majority of such variation is related to origin of this vessel (e.g. from aortic arch or thyrocervical trunk [5]). Absence of the VA appears to be a rare anomaly [1], but may be more common 


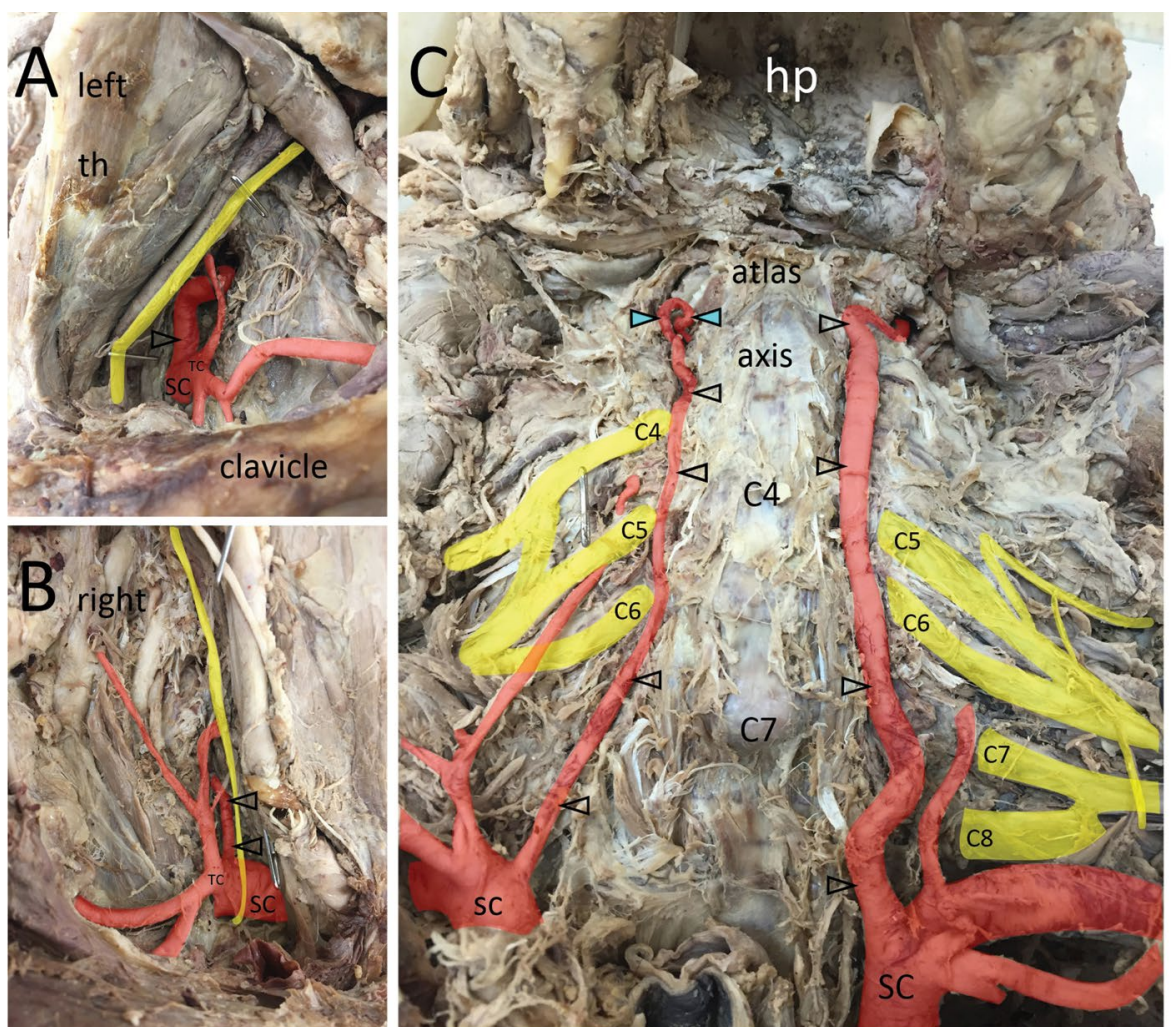

Figure 1. Origin of the anomalous vertebral artery (VA). The origin of the left VA (arrowhead) is shown in panel A (root of the neck), and slightly enlarged (th — thyrohyoid muscle; SC — subclavian artery; TC — thyrocervical trunk). The vagus nerve is indicated in yellow. The origin of the right VA is shown in panel B (see arrowheads); here the VA has a smaller than normal diameter. The sympathetic chain is indicated in yellow. In panel C, the tongue, floor of the oral cavity, pharynx, larynx, and oesophagus have been removed and the transverse processes have been opened to show the full course of the VAs (arrowheads; hp - hard palate). The right VA was notably smaller than the left. As the right VA exited the transverse foramen of the axis, it demonstrated a distinct tortuosity (blue arrowheads) before entering the transverse foramen of the atlas. The roots of the brachial plexus are indicated in yellow.

in individuals with cranio-vertebral anomalies [17]. Regardless, variations in the vascularisation of the brain are clinically important and awareness of such anomalies is crucial for proper diagnosis and treatment of cranio-vertebral and brain disorders.

\section{CASE REPORT}

During brain removal on a 79-year-old male anatomical donor, we observed an enlarged left VA (> $5 \mathrm{~mm}$ in diameter [13]) which continued along to the anterior aspect of the pons, without receiving a VA from the right side. The cause of death was documented to be from a stroke. The head and neck from this specimen was subsequently preserved for detailed dissection at a later time.

Dissection of root of the neck in this specimen revealed VAs arising from the first segment of the subclavian artery bilaterally (Fig. 1). The outer diameters of the subclavian and vertebral arteries in this subject are summarised in Table 1. The first segment
Table 1. Summary of arterial diameters

\begin{tabular}{lcc}
\hline Vertebral artery & Left side $[\mathrm{mm}]$ & Right side $[\mathrm{mm}]$ \\
\hline Posterior fossa & 6.08 & 1.90 \\
C2 & 5.34 & 2.66 \\
C4 & 5.00 & 2.56 \\
C6 & 4.10 & 2.70 \\
Origin & 5.68 & 2.88 \\
Subclavian artery & 11.80 & 10.00 \\
\hline
\end{tabular}

of the subclavian artery measured $11.80 \mathrm{~mm}$ in outer diameter on the left and $10 \mathrm{~mm}$ on the right (Fig. 1C). At the origin, the VA measured $5.68 \mathrm{~mm}$ in outer diameter on the left and $2.88 \mathrm{~mm}$ on the right (Fig. 1). Both VAs entered the transverse foramen of the $6^{\text {th }}$ cervical vertebra (C6); here the VA measured $4.1 \mathrm{~mm}$ on the left and $2.7 \mathrm{~mm}$ on the right (Fig. 1C). Both VAs ascended towards the skull through the transverse foramina. At $\mathrm{C} 4$, the VAs measured $5 \mathrm{~mm}$ in diameter 

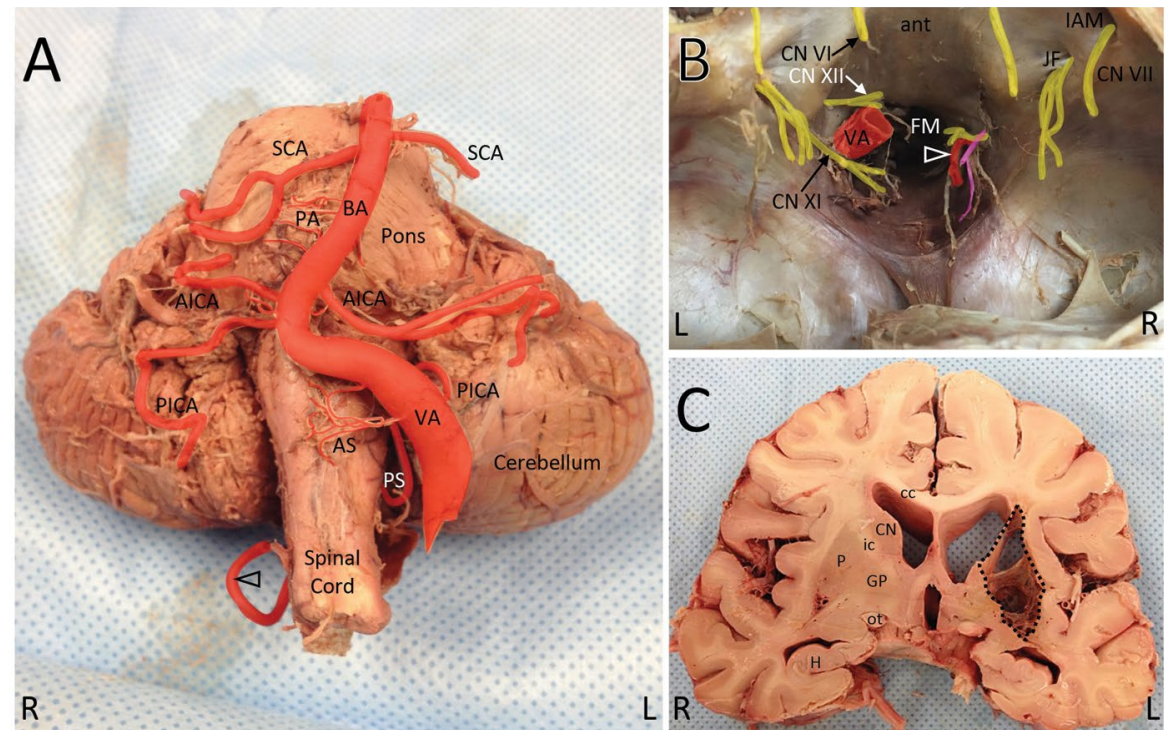

Figure 2. Intracranial distribution of vertebral arteries (VAs). The hindbrain is shown in panel A, with the posterior circulation intact. The arteries have been coloured in red to highlight their distribution. The left VA was noticeably enlarged in the posterior fossa and gave rise to all vessels supplying the medulla oblongata, cerebellum and pons (PS — posterior spinal artery; AS — anterior spinal; PICA — posterior inferior cerebellar; AICA — anterior inferior cerebellar; PA — pontine arteries; BA — basilar artery; SCA — superior cerebellar; R — right; L — left). The right VA (black arrowhead) supplied only the posterior aspect of the spinal cord. The posterior fossa is shown in panel $\mathbf{B}$. The VAs are highlighted in red and cranial nerves in yellow (CN VI — abducens; CN XII — hypoglossal; CN VII - facial; CN XI — spinal accessory; IAM — internal auditory meatus; $\mathrm{FM}$ — foramen magnum; JF — jugular foramen). A meningeal branch of the ascending pharyngeal artery (traversing the hypoglossal canal) is highlighted in pink. The right VA (white arrowhead) was directed posteriorly as it emerged into the posterior cranial fossa (compare to panel A). Coronal slicing of the forebrain revealed the image shown in panel C. A large stroke is indicated by the black dashed line (cc - corpus callosum; $\mathrm{CN}$ — caudate nucleus; ic — internal capsule; $\mathrm{P}$ — putamen; GP — globus pallidus; ot — optic tract; $\mathrm{H}$ - hippocampus).

on the left and $2.56 \mathrm{~mm}$ on the right. At C2, the VAs measured $5.34 \mathrm{~mm}$ in diameter on the left and 2.66 $\mathrm{mm}$ on the right. On the left side, the VA exited the transverse foramen of $\mathrm{C} 2$, turned and ran horizontally to reach the transverse foramen of the atlas. On the right side, the VA exited the transverse foramen of $\mathrm{C} 2$ and demonstrated a distinct tortuosity, forming a complete arterial loop before entering the transverse foramen of the atlas (Fig. 1C, blue arrowheads).

Inside the skull, the left VA was enlarged, measuring $6.08 \mathrm{~mm}$ in diameter (Fig. 2 [13]). This vessel continued to course superiorly along the medulla oblongata and pons, and gave rise to branches typical of the vertebral and basilar arteries. As the basilar artery coursed along the basilar pons, it was situated to the left of the midline. In the posterior fossa, the right VA measured $1.9 \mathrm{~mm}$ in diameter (Fig. 2A, arrowhead). Rather than being directed towards the anterior aspect of the medulla oblongata, this vessel coursed posteriorly (Fig. 2B, white arrowhead). The right VA provided terminal branches to the posterior aspect of the spinal cord, consistent with the territory of the posterior spinal artery. Finally, on coronal sectioning of the brain, a recent infarct was noted to involve the caudate nucleus, posterior limb of the internal capsule, globus pallidus and putamen on the left side (Fig. 2C, black dashed line).

\section{DISCUSSION}

A thorough understanding of anatomical variation, no matter how rare, is essential for accurate diagnosis and treatment of patients. Indeed, it is estimated that a significant number of malpractice cases are linked to inadequate knowledge of anatomical variation [11]. The VAs are associated with a number of anatomical variations. For example, in approximately $4-6 \%$ of subjects the left VA arises from the aortic arch $[5,11]$. Additionally, in nearly $60-70 \%$ of subjects, the VAs are of unequal size, with the left more commonly larger than the right $[6,13,15]$. More rarely, a single VA is present, or one VA fails to reach the contralateral vessel to form the basilar artery. For example, a subject with a single right intracranial VA giving rise to the basilar artery was recently reported [14]. In this subject the left VA arose from the subclavian artery in the neck and terminated by supplying deep neck musculature. Furthermore, there have been two previous reports of 
right VAs reaching the posterior cranial fossa and supplying the cerebellum, but failing to join the left VA $[2,3]$. According to our literature searches, this is only the third published report on the absence of the intracranial portion of the right VA. However, this case is unique in that the right VA enters the posterior cranial fossa, provides arterial supply to the spinal cord, but fails to supply the cerebellum or join the VA from the contralateral side.

In this subject, the right VA supplied the spinal cord, terminating as a posterior spinal artery. Thus, the left VA provided the sole origin of the basilar artery, and the basilar artery in this subject gave rise to both left and right posterior inferior cerebellar arteries. Therefore, the left VA provided arterial support to the entire medulla, cerebellum, pons, midbrain, thalamus and occipital lobe of the cerebral cortex. A blockage in the left VA in this subject would deprive the entire territory of the posterior circulation, because of the lack of collateral supply from the right VA. Additionally, the intracranial left VA was larger in diameter than at C2 (Table 1). The reason for this in unclear, but might be related to altered intracranial blood flow patterns, such as backward flow from the anterior circulations. Such an enlargement might lead to compression of the medulla oblongata, cranial nerves in the posterior cranial fossa and/or cerebellum [8].

In addition to the right VA being shortened/hypoplastic (less than $2 \mathrm{~mm}$ in diameter [16]) and there being marked diameter asymmetry in this subject, this artery had a tortuous segment which formed a complete loop between the axis and atlas. Such a vascular anomaly might be at risk of iatrogenic injury during manual therapy, trauma, positioning for surgery and removal of tumours at the base of the skull. Indeed, a previous study showed that tortuosity of the VA is associated with increased risk of arterial dissection and iatrogenic injury [9].

Anomalies of the VA can cause disruptions in vertebrobasilar blood supply and put the patient at risk of serious cerebrovascular events. Alterations in cerebrovascular haemodynamics may allow for turbulent blood flow, increasing the patient's likelihood of a stroke [7, 12]. In fact, hypoplasia of VA has been associated with increased risk of an ischaemic event $[4,10,12]$. Moreover, lateral displacement of the basilar artery likely contributed to vertebra-basilar insufficiency in this subject $[2,7]$. In this particular subject's brain, altered cerebral haemodynamics may have put him at a higher risk for the infarct found in the internal capsule. In having only one VA, this could have left the patient with insufficient blood flow to various parts of the brain, leaving some areas underperfused. We did not examine the middle cerebral artery in this subject for pathology (which would have supplied the infarcted area). Regardless, it is unclear to what degree the missing VA contributed to the infarct in this subject.

\section{Acknowledgements}

The authors would like to thank the donor and his family for their generous donation.

\section{REFERENCES}

1. Adachi B. Das Arteriensystem der Japaner. Vol. I. Maruzen, Kyoto, Tokyo 1928.

2. Batujeff N. Eine seltene arterienanomalie. Ursprung der a basilaris aus der a carotis internal. Anat Anz. 1889; 4: 282.

3. Berry RJ. case of nonunion of the vertebrales with consequent abnormal origin of the basilaris. Anat Anz. 1910; 35: 54-65.

4. Chuang YM, Huang YC, Hu HH, et al. Toward a further elucidation: role of vertebral artery hypoplasia in acute ischemic stroke. Eur. Neurol. 2006; 55(4): 193-197, doi: 10.1159/000093868, indexed in Pubmed: 16772715.

5. Ergun O, Gunes Tatar I, Birgi E, et al. Evaluation of vertebral artery dominance, hypoplasia and variations in the origin: angiographic study in 254 patients. Folia Morphol. 2016; 75(1): 33-37, doi: 10.5603/FM.a2015.0061, indexed in Pubmed: 26365867.

6. Giuffrè R, Sherkat S. Maldevelopmental pathology of the vertebral artery in infancy and childhood. Childs Nerv Syst. 2000; 16(10-11): 627-632, doi:10.1007/PL00013718, indexed in Pubmed: 11151711.

7. Hong JM, Chung CS, Bang OY, et al. Vertebral artery dominance contributes to basilar artery curvature and peri-vertebrobasilar junctional infarcts. J Neurol Neurosurg Psychiatr. 2009; 80(10): 1087-1092, doi: 10.1136/ jnnp.2008.169805, indexed in Pubmed: 19414436.

8. Krayenbuhl $\mathrm{H}$, Yasargil MG. [The vascular diseases in the region of the basilar and vertebral arteries; an anatomopathological, clinical and neuroradiological study]. Fortschr Geb Rontgenstr Nuklearmed. 1957; 87(Ergbd. 80): 1-170, indexed in Pubmed: 13462004.

9. Morris SA, Orbach DB, Geva T, et al. Increased vertebral artery tortuosity index is associated with adverse outcomes in children and young adults with connective tissue disorders. Circulation. 2011; 124(4): 388-396, doi: 10.1161/CIRCULATIONAHA.110.990549, indexed in Pubmed: 21730308.

10. Perren F, Poglia D, Landis T, et al. Vertebral artery hypoplasia: a predisposing factor for posterior circulation stroke? Neurology. 2007; 68(1): 65-67, doi: 10.1212/01.wnl.0000250258.76706.98, indexed in Pubmed: 17200496.

11. Sańudo J, Vázquez R, Puerta J. Meaning and clinical interest of the anatomical variations in the 21 st century. Eur J Anat. 2003; 7(suppl. 1): 1-3. 
12. Satti SR, Cerniglia CA, Koenigsberg RA. Cervical vertebral artery variations: an anatomic study. AJNR Am J Neuroradiol. 2007; 28(5): 976-980, indexed in Pubmed: 17494682.

13. Seidel E, Eicke BM, Tettenborn B, et al. Reference values for vertebral artery flow volume by duplex sonography in young and elderly adults. Stroke. 1999; 30(12): 2692-2696, doi: 10.1161/01.str.30.12.2692, indexed in Pubmed: 10582999.

14. Shoja M, Tubbs $R$, Khaki $A$, et al. rare variation of the vertebral artery. Folia Morphol. 2006; 65: 167-170.

15. Tetiker H, Çimen M, Kosar M. Evaluation of the Vertebral Artery by 3D Digital Subtraction Angiography. Interna- tional Journal of Morphology. 2014; 32(3): 798-802, doi: 10.4067/s0717-95022014000300010.

16. Thierfelder KM, Baumann AB, Sommer WH, et al. Vertebral artery hypoplasia: frequency and effect on cerebellar blood flow characteristics. Stroke. 2014; 45(5): 1363-1368, doi: 10.1161/STROKEAHA.113.004188, indexed in Pubmed: 24699051.

17. Wang S, Wang C, Liu Yi, et al. Anomalous vertebral artery in craniovertebral junction with occipitalization of the atlas. Spine. 2009; 34(26): 2838-2842, doi: 10.1097/BRS.0b013e3181b4fb8b, indexed in Pubmed: 20010391. 